\title{
Injectable calcium phosphate with hydrogel fibers encapsulating induced pluripotent, dental pulp and bone marrow stem cells for bone repair
}

\author{
Lin Wang ${ }^{\mathrm{a}, \mathrm{b}}$, Chi Zhang ${ }^{\mathrm{b}, \mathrm{c}}$, Chunyan $\mathrm{Li}^{\mathrm{a}}$, Michael D. Weir ${ }^{\mathrm{b}}$, \\ Ping Wang ${ }^{b^{*}}$, Mark A. Reynolds ${ }^{b}$, Liang Zhao ${ }^{\text {b,d** }}$, Hockin H. K. Xu ${ }^{\text {be, f }}$ \\ ${ }^{a}$ VIP Integrated Department, School and Hospital of Stomatology, Jilin University, \\ Changchun, Jilin 130011,China \\ ${ }^{\mathrm{b}}$ Department of Endodontics, Periodontics and Prosthodontics, University of Maryland \\ School of Dentistry, Baltimore, MD 21201, USA \\ ${ }^{c}$ State Key Laboratory of Oral Diseases, West China School of Stomatology, Sichuan \\ University, Chengdu, 610041 China \\ ${ }^{\mathrm{d}}$ Department of Orthopaedic Surgery, Nanfang Hospital, Southern Medical University, \\ Guangzhou, Guangdong 510515, China \\ ${ }^{\mathrm{e}}$ Center for Stem Cell Biology \& Regenerative Medicine, University of Maryland School of \\ Medicine, Baltimore, MD 21201, USA \\ ${ }^{\mathrm{f}}$ Department of Mechanical Engineering, University of Maryland Baltimore County, \\ Baltimore County, MD 21250, USA
}

For: Materials Science and Engineering: C (Materials for Biological Applications)

(Submitted in April 2016; revised and resubmitted in Jun 2016;

$2^{\text {nd }}$ revised and resubmitted in Jul 2016)

Correspondence: Dr. Ping Wang, University of Maryland School of Dentistry, Baltimore, MD 21201, email: pwang@umaryland.edu. Dr. Liang Zhao, Department of Orthopaedic Surgery, Southern Medical University, Guangzhou, Guangdong 510515, China, email: lzhaonf@126.com.

Short title: Injectable CPC with hydrogel fibers encapsulating stem cells 
Key words: calcium phosphate cement, human induced pluripotent stem cells, dental pulp stem cells, bone marrow stem cells, cell-encapsulating fibers.

\begin{abstract}
Human induced pluripotent stem cell-derived mesenchymal stem cells (hiPSC-MSCs), dental pulp stem cells (hDPSCs) and bone marrow MSCs (hBMSCs) are exciting cell sources in regenerative medicine. However, there has been no report comparing hDPSCs, hBMSCs and hiPSC-MSCs for bone engineering in an injectable calcium phosphate cement (CPC) scaffold. The objectives of this study were to: (1) develop a novel injectable CPC containing hydrogel fibers encapsulating stem cells for bone engineering, and (2) compare cell viability, proliferation and osteogenic differentiation of hDPSCs, hiPSC-MSCs from bone marrow (BM-hiPSC-MSCs) and from foreskin (FS-hiPSC-MSCs), and hBMSCs in CPC for the first time. The results showed that the injection did not harm cell viability. The porosity of injectable CPC was 62\%. All four types of cells proliferated and differentiated down the osteogenic lineage inside hydrogel fibers in CPC. hDPSCs, BM-hiPSC-MSCs, and hBMSCs exhibited high alkaline phosphatase, runt-related transcription factor, collagen I, and osteocalcin gene expressions. Cell-synthesized minerals increased with time $(p<0.05)$, with no significant difference among hDPSCs, BM-hiPSC-MSCs and hBMSCs $(p>0.1)$. Mineralization by hDPSCs, BM-hiPSC-MSCs, and hBMSCs inside CPC at $14 \mathrm{~d}$ was 14-fold that at $1 \mathrm{~d}$. FS-hiPSC-MSCs were inferior in osteogenic differentiation compared to the other cells. In conclusion, hDPSCs, BM-hiPSC-MSCs and hBMSCs are similarly and highly promising for bone tissue engineering; however, FS-hiPSC-MSCs were relatively inferior in osteogenesis. The novel injectable CPC with cell-encapsulating hydrogel fibers may enhance
\end{abstract}


bone regeneration in dental, craniofacial and orthopedic applications.

\section{Introduction}

Bone defects with limited intrinsic regenerative potential represent a considerable surgical challenge and are associated with high socio-economical costs and severe reduction on patients' quality of life. In the United States alone, over one million bone grafting, bone excision and fracture repair surgeries are performed annually costing approximately $\$ 5$ billions [1]. Bone tissue engineering involving the use of scaffolds, cells, and bioactive factors is promising to meet this tremendous need [2]. Stem cells have been extensively studied and cell-based tissue engineering represents a new approach in regenerative medicine [3-7]. Human bone marrow mesenchymal stem cells (hBMSCs), as a type of postnatal stem cells, are multipotent and able to differentiate into osteoblasts, chondrocytes, neurons, myoblasts, adipocytes, and fibroblasts [8]. hBMSCs are currently regarded as the gold standard cell source for many tissue-engineering therapies and have been successfully applied in clinics $[9,10]$. However, autogenous hBMSCs require an invasive procedure to harvest and are limited in cell numbers, and the cell potency decreases due to aging and diseases [11]. Therefore, alternative stem cell sources are needed for bone regeneration.

Recently, human induced pluripotent stem cells (hiPSCs) were generated from adult somatic cells using reprogramming techniques, which represented a major breakthrough [12]. iPSCs provide a promising strategy to obtain patient-specific stem cells for tissue engineering. In order to reduce the risk of tumorigenesis of hiPSCs, MSCs were derived from hiPSCs (referred to as hiPSC-MSCs) [13]. hiPSC-MSCs exhibited a higher proliferative capability 
than BMSCs [14], and were less tumorigenic than undifferentiated iPSCs [15]. Another issue concerning iPSCs' clinical application is whether the type of original somatic cells has any influence on the properties of the generated iPSCs. Evidence showed that iPSCs retained, at least transiently, epigenetic memory related to the donor cell of origin, which might affect their subsequent differentiation potential [16-19]. Therefore, two distinct hiPSCs, one from bone marrow CD34+ mononuclear cells and the other from foreskin fibroblasts, were included in this study to compare their osteogenic potential.

Human dental pulp stem cells (hDPSCs), as another postnatal stem cells, share similar gene expression profiles and differentiation capability to that of BMSCs [20,21]. The most striking feature of DPSCs is their ability to regenerate a dentin-pulp-like complex that is composed of mineralized matrix with tubules lined with odontoblasts, and fibrous tissue containing blood vessels in an arrangement similar to the dentin-pulp complex found in normal human teeth [21]. Previous studies showed that DPSCs, when undergoing differentiation to pre-osteoblasts, deposited an extracellular matrix which became a calcified woven bone tissue [22]. DPSCs are potentially superior to other stem cells as teeth are easy to access. Therefore, DPSCs are promising for use in bone tissue engineering.

Calcium phosphates $(\mathrm{CaP})$ are wildly used for bone repair because of their similarities to the inorganic components of bone [23]. However, for traditional pre-formed scaffolds to fit in a bone cavity, surgeons need to machine the graft or carve the surgical site, leading to increases in bone loss, trauma and surgical time [24]. These scaffolds for cell delivery have drawbacks including the difficulty of seeding cells deep into the scaffold, and inability for injection in minimally invasive surgeries [25]. Therefore, there is a need for the development 
of injectable bone substitutes suitable for cell delivery and minimally invasive surgeries.

In the present study, cells were encapsulated in alginate-fibrin hydrogel fibers, and these cell-loaded fibers were then mixed with CPC paste to form an injectable construct. This strategy provided the protection for cells during CPC mixing, injection and setting reaction, prolonged the retention of the delivered cells at the graft site and offered a physiological three-dimensional environment for the seeded cells. In addition, fibers can provide more space for cellular self-assembly through which living cells may organize into functional units, allowing the cells to grow, migrate and differentiate in the extracellular matrix [26].

The objectives of this study were to: (1) develop a novel injectable CPC construct containing hydrogel fibers encapsulating stem cells for bone engineering, and (2) investigate cell viability, proliferation and osteogenic differentiation by comparing hDPSCs, BM-hiPSC-MSCs, FS-hiPSC-MSCs and hBMSCs in CPC for the first time. The following hypotheses were tested: (1) the injection process of CPC would not harm the encapsulated cells; (2) the injectable cell-encapsulating CPC would have fast setting time and high porosity; (3) BM-hiPSC-MSCs would be superior in osteogenesis to FS-hiPSC-MSCs; (4) Both BM-hiPSC-MSCs and hDPSCs would be viable alternative cell sources for bone tissue engineering, matching the osteogenic potential of the gold-standard hBMSCs.

\section{Materials and Methods}

\subsection{Culture, derivation and immunophenotype of hiPSC-MSCS}

The culture of hiPSCs was approved by the University of Maryland Institutional Review Board (HP-00046649). hiPSC BC1 line derived from adult bone marrow CD34+ cells (BM-iPSCs) and hiPSC (Foreskin) Clone 1 derived from human foreskin fibroblast 
(FS-iPSCs) were used. BM-iPSCs were derived from adult bone marrow CD34+ cells which were reprogramed by a single episomal vector pEB-C5, as previously described [27]. BM-iPSCs were maintained on mitotically-inactivated murine embryonic fibroblasts (MEF) feeder cells in hiPSC medium [27]. The hiPSC medium consisted of $80 \%$ Dulbecco's modified Eagle medium (DMEM)/F12 (Invitrogen, Carlsbad, CA), 20\% Knockout Serum Replacement (a serum-free formulation, Invitrogen), 1\% MEM non-essential amino acids solution, $10 \mathrm{ng} / \mathrm{mL}$ basic fibroblast growth factor ( $\beta$-FGF, Invitrogen), $1 \mathrm{mM}$ L-glutamine (Sigma) and $0.1 \mathrm{mM} \beta$-mercaptoethanol (Sigma). BM-hiPSCs were detached from a feeder layer and dissociated into clumps through treatment with $1 \mathrm{mg} / \mathrm{mL}$ collagenase type IV in DMEM/F12 at $37{ }^{\circ} \mathrm{C}$ for $6 \mathrm{~min}$, followed by mechanical scraping. The dissociated BM-hiPSC clumps were collected by sedimentation, re-suspended in embryoid body (EB) differentiation medium (the same formulation as hiPSC culture medium but without $\beta$-FGF), and transferred to $25 \mathrm{~cm}^{2}$ ultra-low attachment cell culture flasks (Corning, Corning, NY) [27].

After 10 days (d), the EBs were transferred onto 0.1\% gelatin-coated culture dishes [27]. Cells growing out from EBs were cultured and upon $70 \%$ confluence, the outgrowth cells (P0) were selectively isolated by using cell scrapers and sub-cultured in MSC growth medium. The MSC growth medium consisted of low glucose DMEM (Gibco, Grand Island, NY) supplemented with $10 \%$ fetal bovine serum (FBS, HyClone, Logan, UT), $100 \mathrm{U} / \mathrm{mL}$ penicillin, and $100 \mathrm{mg} / \mathrm{mL}$ streptomycin (PS, Gibco). The differentiated cells from these culture conditions were passaged until a homogeneous fibroblastic morphology appeared. They were termed BM-hiPSC-derived MSCs (BM-hiPSC-MSCs) [27]. Our previous study 
confirmed that the hiPSC-MSCs generated from this method expressed surface markers characteristic of MSCs (CD29, CD44, CD166, CD73), and were negative for typical hematopoietic (CD34), endothelial (CD31) and pluripotent markers (TRA-1-81 and OCT 3/4) [28]. The 4th passage hiPSC-MSCs were used in subsequent experiments.

FS-hiPSCs were purchased from WiCell (Madison WI, USA). Feeder-free culture condition has been developed for undifferentiated iPSCs by the WiCell. The FS-iPSCs were cultured on $0.1 \%$ Matrigel coated plate in mTeSR 1 medium in a feeder-free condition. The medium was changed daily. FS-hiPSCs were detached from a Matrigel layer and dissociated into clumps through treatment with $1 \mathrm{mg} / \mathrm{mL}$ collagenase type IV in DMEM/F12 at $37{ }^{\circ} \mathrm{C}$ for 6 min, followed by mechanical scraping. The dissociated FS-hiPSC clumps were collected by sedimentation, re-suspended in embryoid body (EB) culture medium, and transferred to $25 \mathrm{~cm}^{2}$ ultra-low attachment cell culture flasks (Corning, Corning, NY). After $10 \mathrm{~d}$ of suspension culture, the same protocol was carried out to derive MSCs from FS-hiPSCs as the BM-hiPSC-MSCs derivation protocol. The differentiated cells from these culture conditions were passaged until a homogeneous fibroblastic morphology appeared. They were termed as FS-hiPSC-derived MSCs (FS-hiPSC-MSCs).

To analyze expression of characteristic mesenchymal protein marker, surface antigens were detected via flow cytometry [29]. FS-hiPSC-MSCs (Passage 4) were harvested by trypsin-ethylenediaminetetraacetic acid (EDTA) and washed with phosphate buffered saline (PBS) containing $1 \%$ bovine serum albumin (BSA), then resuspended to $1 \times 10^{6}$ cells in $50 \mu \mathrm{L}$ of cold PBS containing 1\% BSA. Cell samples were separately labeled on ice with optimal dilution of fluorescein isothiocyanate (FITC)-conjugated monoclonal antibodies (mAbs, from 
Invitrogen, unless otherwise noted) against CD29, CD31, CD34 (BD Pharmingen, San Jose, CA), CD44, CD73, CD105, TRA-1-81, HLA-ABC and HLA-DR, phycoerythrin (PE)-conjugated mAbs against CD166, and Alexa Fluor 488 conjugated mAb against CD105 in the dark. After 30 min incubation, cells were washed with cold PBS. Non-specific fluorescence was determined by incubating cells with isotype-matched conjugated mAbs. At least 10,000 events were collected from each run of flow cytometry. Data were analyzed using CellQuest software (Becton, San. Jose, CA). A fluorescence histogram for each mAb was displayed alongside the control antibody. The percentages of positive cells were subtracted from the isotype control antibody of each conjugate [29].

\subsection{Isolation and culture of hDPSCs}

Human dental pulp stem cells (hDPSCs) were obtained from pulps of healthy teeth from 14-22 year old patients who had their teeth extracted due to orthodontic treatment. The procedure was reviewed and approved by the Institutional Review Board of the University of Maryland Baltimore School of Dentistry. Cells were isolated as described previously [30]. In brief, pulp tissues were minced and digested in a solution of $3 \mathrm{mg} / \mathrm{mL}$ of collagenase type I and $4 \mathrm{mg} / \mathrm{mL}$ dispase for $30-60 \mathrm{~min}$ at $37^{\circ} \mathrm{C}$. Cell suspension was obtained by passing the digested tissue through a $70-\mu \mathrm{m}$ cell strainer. The cells were pelleted and seeded in culture dishes, and incubated with DMEM growth medium (DMEM + 10\% FBS + 1\% PS, Gibco) in a humidified atmosphere of $95 \%$ air and $5 \% \mathrm{CO}_{2}$. Non-adherent cells were removed 48 hours (h) after the initial plating. The medium was replaced every $3 \mathrm{~d}$. When primary culture became subconfluent, after approximately 1-2 weeks, cells were collected by trypsinization and subcultured at 5,000 cells per $\mathrm{cm}^{2}$ in growth medium. Previous study confirmed that the 
hDPSCs generated from this method expressed surface markers characteristic of MSCs (CD29, CD44, CD166, CD73), and were negative for typical hematopoietic (CD34, CD45, CD14) [30]. The 4th passage hDPSCs were used in the following experiments.

\section{3 hBMSCs culture}

hBMSCs were commercially obtained from Lonza (Allendale, NJ). hBMSC were maintained in DMEM growth medium (Gibco). Routine characterization of hBMSCs was provided by the manufacture. It included testing for surface antigens and functional testing for differentiation down specific lineage pathways. Cells were tested positive for CD105, CD166, CD29 and CD44, but negative for CD14, CD34, CD45 by flow cytometry. The 4th passage hBMSCs were used in the following experiments.

\subsection{Cell encapsulation in alginate-fibrin fibers}

Alginate has been used for cell delivery because it can be ionically cross-linked with multivalent cations under mild conditions without harming the cells, and alginate hydrogels are highly hydrated with good biocompatibility [31]. However, alginate degrades slowly and uncontrollably. A previous study developed a method to control alginate gel degradation via partial periodate oxidation [32]. In our previous study, alginate with $7.5 \%$ oxidation was verified to possess the ability of fast cell release from microbeads [33]. Therefore, in the present study, alginate (64\% guluronic acid, MW = 75,000-220,000 g/mol, ProNova, Oslo, Norway) was oxidized at $7.5 \%$ oxidation as previously described [33]. Cell-encapsulating alginate fibers were synthesized using a wet spinning technique, which consisted of the extrusion of a solution through a needle into a coagulation bath [34]. A $2 \%$ sodium alginate 
solution was prepared by dissolving alginate in a $155 \mathrm{mM}$ sodium chloride solution at $37{ }^{\circ} \mathrm{C}$. This was followed by adding fibrinogen from bovine plasma (Sigma) at a concentration of $0.4 \%$ [35]. Cells were added to the alginate-fibrinogen solution at a density of $1 \times 10^{6}$ cells $/ \mathrm{mL}$. The cell-alginate suspension was loaded into a $5 \mathrm{~mL}$ syringe. The cell-encapsulating alginate-fibrin fibers were obtained by extruding the cell-alginate suspension through a 27-gauge needle (with $210 \mu \mathrm{m}$ inner diameter) via a syringe pump (NE-300, New Era Pump Systems, Farmingdale, NY) at $6 \mathrm{~mL} / \mathrm{min}$. The suspension was extruded into a $100 \mathrm{~mL}$ bath which consisted of $100 \mathrm{mmol} / \mathrm{L}$ calcium chloride (Sigma) and $1 \mathrm{NIH}$ units per $\mathrm{mL}$ of thrombin (Sigma). When the alginate-fibrinogen streamed into the bath, the presence of $\mathrm{Ca}^{2+}$ ions initiated the ionotropic gelation by counterbalancing the negative charges of alginate, while the reaction between fibrinogen and thrombin produced fibrin $[33,34]$. The alginate-fibrin fibers were allowed to cross-link in the bath for $20 \mathrm{~min}$ [34]. Then the alginate-fibrin fibers were collected and washed with phosphate buffered saline (PBS). This method resulted in the cell-encapsulating alginate-fibrin fibers. These fibers were examined via a light microscope (TE2000-S, Nikon, Melville, NY). The fiber formed in the cross-linking bath was a single continuous fiber. During handling and for the ease of mixing with CPC paste, the long fiber was cut into short fibers with a length of $15 \mathrm{~mm}$, as described in the following section. These cell-encapsulating alginate-fibrin fibers were referred to as "CAF".

\subsection{Physical properties of CPC pastes containing cell-encapsulating fibers}

CPC powder consisted of tetracalcium phosphate [TTCP: $\mathrm{Ca}_{4}\left(\mathrm{PO}_{4}\right)_{2} \mathrm{O}$ ] and dicalcium 
phosphate anhydrous (DCPA: $\mathrm{CaHPO}_{4}$ ) [24]. TTCP was synthesized using DCPA and calcium carbonate (both from J.T. Baker, Philipsburg, NJ) which were mixed and heated at $1500{ }^{\circ} \mathrm{C}$ for $6 \mathrm{~h}$ in a furnace (Model 51333, Lindberg, Watertown, WI). The heated mixture was quenched to room temperature, ground in a ball mill (Retsch PM4, Brinkman, NY) and sieved to obtain TTCP powder with a median particle size of $5 \mu \mathrm{m}$. The commercial DCPA powder was ground for $24 \mathrm{~h}$ in the ball mill and sieved to obtain a median particle size of 1 $\mu \mathrm{m}$. Then the TTCP and DCPA powders were mixed at 1:3 molar ratio in a micro-mill (Bel-Alert Products, Pequannock, NJ) to form the CPC powder. Chitosan could render the CPC fast-setting and mechanically strong [36]. Therefore, chitosan lactate (Vanson, Redmond, WA) was mixed with water at chitosan/(chitosan + water) mass fractions of $15 \%$ to serve as CPC liquid [24]. The CAF were cut into lengths of approximately $15 \mathrm{~mm}$ and mixed with CPC paste at a fiber volume fraction of 50\%. The CAF were flexible and were randomly bent and oriented in the CPC paste. For mechanical reinforcement, an absorbable suture fiber (Vicryl, polyglactin 910, Ethicon, Somerville, NJ) was cut to $3 \mathrm{~mm}$ length and mixed in CPC paste at $10 \%$ by volume, which could provide several weeks of early mechanical strength to the CPC scaffold [37]. Therefore, four CPC pastes were tested for injectability (Chitosan is referred to as $\mathrm{CN}$, and suture fiber is referred to as $\mathrm{SU})$ :

(1) CPC paste using distilled water as liquid, 0\% chitosan (referred to as CPC control);

(2) $\mathrm{CPC}+50 \%$ cell-encapsulating alginate-fibrin fibers (referred to as CPC-CAF);

(3) $\mathrm{CPC}+15 \%$ chitosan liquid $+50 \%$ cell-encapsulating alginate-fibrin fibers (CPC-CN-CAF);

(4) $\mathrm{CPC}+15 \%$ chitosan liquid $+50 \%$ cell-encapsulating alginate-fibrin fibers $+10 \%$ 
suture fiber (CPC-CN-CAF-SU);

Cement setting time was measured using a method described in a previous study [38]. CPC paste was mixed at a powder:liquid ratio of $2: 1$, filled into a mold of $3 \times 4 \times 25 \mathrm{~mm}$, and placed in a humidor at $37^{\circ} \mathrm{C}$. At one-minute intervals, the specimen was scrubbed gently with fingers until the powder component did not come off, indicating that the setting reaction had occurred sufficiently to hold the specimen together. The time measured from the powder-liquid mixing to this point was used as the setting time (mean $\pm \mathrm{sd} ; n=6$ ) [38].

To measure porosity, CPC specimens of $3 \times 4 \times 25 \mathrm{~mm}$ were incubated at $37^{\circ} \mathrm{C}$ in distilled water for $24 \mathrm{~h}$, and then dried in a vacuum oven at $60^{\circ} \mathrm{C}$ for $24 \mathrm{~h}$. The dried specimens were placed in the chamber of a porosimeter (PoreMaster GT; Quantachrome, Boynton Beach, FL, USA). The chamber containing the specimens was gradually filled with mercury up to a pressure of $210 \mathrm{MPa}$. The known chamber volume, mercury density and specimen weight enabled the specimen's volume, density and porosity to be calculated (mean $\pm \mathrm{sd} ; n=6$ ) [38].

To examine the alginate fibers and CPC particles in the constructs, six aforementioned specimens of CPC-CN-CAF were used for scanning electron microscope (SEM; FEI Quanta 200, Hillsboro, OR, USA) examination. Specimens were immersed in distilled water for 1 day for complete setting of CPC. After dehydration, both the external surfaces and interior cross-sections of the specimens were sputter-coated with gold and examined in SEM.

\subsection{Viability of encapsulated hDPSCs. BM-hiPSC-MSCs, FS-hiPSC-MSCs and hBMSCs}

hDPSCs, BM-hiPSC-MSCs, FS-hiPSC-MSCs and hBMSCs were each encapsulated in alginate-fibrin fibers. To evaluate if the CPC mixing and injection would harm the 
encapsulated cells and to compare the viability of hDPSCs, BM-hiPSC-MSCs, FS-hiPSC-MSCs and hBMSCs, cell viability was examined without injection and after injection in a CPC-CN-SU paste. The paste was injected from a $10 \mathrm{~mL}$ syringe (Free-Flo, Kerr, Romulus, MI) with an opening tip of $2.7 \mathrm{~mm}$ which is similar to the inner diameter of a 10-gauge needle [24]. The 10-gauge needle was similar to spinal needles used in augmentation of osteoporotic vertebrae and the management of vertebral compression fractures. After CPC powder and liquid (2:1 mass ratio) were mixed, the paste was immediately placed into the syringe and inject manually. Then, the CPC paste was washed by $100 \mathrm{mM} \mathrm{CaCl}{ }_{2}$ completely, and then the CAF were collected. The CAF before and after injection were stained with a live/dead kit (Molecular Probes, Eugene, OR). The CAF were placed into a 6-well plate and incubated with $4 \mu \mathrm{M}$ ethidium homodimer-1 (EthD-1) and 2 $\mu \mathrm{M}$ calcein-AM in PBS for $20 \mathrm{~min}$. The CAF were examined using epifluorescence microscope (Eclipse TE2000-S, Nikon, Melville, NY). The percentage of live cells $P$ and the live cell density $D$ were measured and calculated as previously described [14]. $\mathrm{P}_{\text {Live }}=$ $\mathrm{N}_{\text {Live }} /\left(\mathrm{N}_{\text {Live }}+\mathrm{N}_{\text {Dead }}\right)$, where $\mathrm{N}_{\text {Live }}$ and $\mathrm{N}_{\text {Dead }}$ were the number of live and dead cells, respectively. $\quad D_{\text {Live }}=\mathrm{N}_{\text {Live }} / A$, where $A$ was the area of the image where $\mathrm{N}_{\text {Live }}$ was measured [14]. Six samples per condition (before and after injection) for each cell type were fabricated for this measurement. Three randomly-chosen images for each sample were analyzed with six samples per condition, yield 18 images per condition for each cell type (technical replicate $n$ $=18)$.

To examine the cell release from $\mathrm{CAF}$ inside the $\mathrm{CPC}$, the $\mathrm{CAF}$ were placed inside the CPC paste, using a sandwich method described previously [39]. First, $0.1 \mathrm{~g}$ of a CPC 
paste was placed to cover the bottom of a well (15 mm diameter) of a 24 -well plate and the CPC paste surface was flattened. Then, $200 \mu \mathrm{L}$ of CAF was placed on the CPC, and another $0.1 \mathrm{~g}$ of CPC paste was used to completely cover the CAF. The purpose of this set-up was to provide a flat bottom CPC surface, so that the cells released from the CAF could attach to CPC to enable live/dead staining and microscopic examination. Ideally, the CAF should be randomly mixed with CPC paste; however, subsequently breaking the CPC scaffold for analysis would create rough and tortuous surfaces unusable for microscopic examination. In the present study, the CAF were completely trapped inside the CPC, enabling the test of cell survival inside CPC, which would rely on the interconnected pores in CPC for fluids and nutrition. The CPC-CAF construct was incubated at $37{ }^{\circ} \mathrm{C}$ for $30 \mathrm{~min}$, then $2 \mathrm{~mL}$ of osteogenic medium ( DMEM growth medium supplemented with $50 \mu \mathrm{g} / \mathrm{ml} \mathrm{L-ascorbic} \mathrm{acid}$ 2-phosphate, and $10^{-8} \mathrm{~mol} / \mathrm{L}$ dexamethasone, Sigma) was added to each well to submerge the construct, which was examined as described below. All of the following cell experiments used the osteogenic medium. At each time points (1, 4, 7 and $14 \mathrm{~d})$, the constructs were opened and the bottom CPC layer was used for analysis. Live/dead staining was performed by immersing the bottom CPC layer in live/dead staining solution (Molecular Probes). For each cell type and each time period, six wells were used for live/dead staining, and six wells were used for cell proliferation assay. The cells were examined via epifluorescence microscopy (Eclipse TE-2000S, Nikon). Three images were taken at random locations for each sample, with 6 samples yielding 18 images for each cell type at each time point. The percentage of live cells $P$ and the live cell density $D$ were measured and calculated as described above. 
In addition, a cell counting kit (CCK-8, Dojindo, Tokyo, Japan) was used to evaluate cell proliferation from 1 to $14 \mathrm{~d}$ in CPC-CAF constructs. Six samples each group was used for cell proliferation assay $(n=6)$. CCK-8 is based on the WST- 8 reaction that produces an orange formazan dye in an amount that is directly related with the number of viable cells [40]. After carefully breaking the CPC cover, the bottom CPC layer was immersed in $2 \mathrm{~mL}$ of culture medium containing $10 \% \mathrm{CCK}-8$, and incubated in dark for $1.5 \mathrm{~h}$. Then $100 \mu \mathrm{L}$ of the medium was transferred to a new 96-well plate. The cell proliferative rate was determined via the absorbance at an optical density of $450 \mathrm{~nm}\left(\mathrm{OD}_{450 \mathrm{~nm}}\right)$ using a microplate reader (SpectraMax M5, Molecular Devices, Sunnyvale, CA), following a previous study [40]. WST-8 was reduced by dehydrogenase activities in cells to give a yellow-color formazan dye, which was soluble in culture medium. The amount of the formazan dye, generated by the activities of dehydrogenases in cells, was directly proportional to the number of living cells [40].

\subsection{Osteogenic differentiation of encapsulated stem cells}

The aforementioned CPC-CAF containing the four types of cells were cultured in osteogenic medium. At 1, 4, 7 and $14 \mathrm{~d}$, the total RNA of the collected cells was extracted with TRIzol reagent and PureLink RNA Mini Kit (Invitrogen), and reverse-transcribed into cDNA using High-Capacity cDNA Reverse Transcription kit (Applied Biosystems, Foster City, CA) in a thermal cycler (GenAmp PCR 2720, Applied Biosystems). TaqMan gene expression kits were used to quantify the targeted genes on human alkaline phosphatase (ALP, Hs00758162_ml), Runt-related transcription factor (RUNX2, Hs00231692_ml), collagen 
type-I, alpha 1 (COL1A1, Hs00164004_m1), osteocalcin (OC, Hs00609452_g1) and glyceraldehyde 3-phosphate dehydrogenase (GAPDH, Hs99999905_m1; reference gene). Relative expression was evaluated using the $2^{-\Delta \Delta \mathrm{Ct}}$ method and normalized by the $\mathrm{C}_{\mathrm{t}}$ of the housekeeping gene GAPDH. $\mathrm{C}_{t}$ of hDPSCs, BM-hiPSC-MSCs, FS-hiPSC-MSCs and hBMSCs cultured on tissue culture polystyrene in control medium at $1 \mathrm{~d}$ served as their own calibrator. The $14 \mathrm{~d}$ culture followed previous studies [24, 39].

\subsection{Mineral synthesis}

CPC-CAF constructs encapsulating the four types of cells were cultured in osteogenic medium. After 1, 7 and $14 \mathrm{~d}$, xylenol orange and Alizarin red S (ARS) staining methods were used to detect mineral synthesis by the encapsulated cells in vitro. For each method, six sample per group were fabricated for mineral synthesis evaluation $(n=6)$.

Xylenol orange, a calcium-chelating fluorochrome, and can stain minerals into a red fluorescent color. It is not harmful to cells and the staining can be performed on live cells [41]. Because CPC could chelate with xylenol orange, the aforementioned sandwich model was modified to avoid a strong background in images. $200 \mu \mathrm{L}$ of CAF was directly placed on the polystyrene bottom of each well of 24-well plate, and then $0.1 \mathrm{~g}$ of CPC paste was used to completely cover the CAF underneath. At 1,7 and $14 \mathrm{~d}$, the CPC crust was removed, the CAF and released cells attached on the culture well was incubated overnight in $2 \mathrm{~mL}$ osteogenic medium containing $100 \mu \mathrm{M}$ xylenol orange. In this way, only mineralized nodules formed in CAF were stained, without interference from CPC $(n=6)$. Mineral nodules were observed via an epifluorescence microscopy (Eclipse TE-2000S, Nikon). The mineral area 
percentage was calculated as $\mathrm{A}_{\text {Mineral }} / \mathrm{A}_{\text {Total }}$, where $\mathrm{A}_{\text {Mineral }}$ is the area of mineralization (red fluorescence) in the image, and $\mathrm{A}_{\text {Total }}$ is the total area of the image [24].

Alizarin red S (ARS) staining was performed on sandwich model constructs as described in 2.6. The CPC bottom layer with the released cells were washed with PBS, fixed in $10 \%$ formaldehyde, and staining with 2\% ARS (Millipore, Billerica, MA) for $30 \mathrm{~min}$, which stained calcium-rich deposits made by cells into a dark red color [39]. An osteogenesis assay kit (Millipore) was used to extract the stained minerals and measure Alizarin red concentration at $\mathrm{OD}_{405 \mathrm{~nm}}$, following the manufacturer's instructions $(n=6)$. The ARS standard curve was made with a known concentration of the dye. CPC without cells served as the control. The control ARS concentration was subtracted from the ARS concentration of constructs with cells to yield the net mineral concentration synthesized by cells [39].

Both xylenol orange and ARS staining methods were also used to detect mineral synthesis by the four types of cells pre-encapsulation. Each type of stem cells was seeded into 6-well plates at a density of $1 \times 105$ per well. Osteogenic medium was added in each well. At 7 and $14 \mathrm{~d}$, xylenol orange and ARS staining methods were used to detect mineral synthesis by the stem cells. For each method, six samples per cell type were tested $(n=6)$.

\subsection{Statistical analyses}

All data were checked for normal distribution with the Kolmogorov-Smirnov test. Testing for significant differences was performed by one-way and two-way analyses of variance (ANOVA) using Tukey's post-hoc test for pairwise comparisons. Statistical analyses were performed by SPSS 19.0 software (SPSS, Chicago, IL) at a pre-set alpha of 0.05. 


\section{Results}

Fig. 1 plots flow cytometry results of FS-hiPSC-MSCs. The MSC surface markers CD29, CD44, CD166, CD73 and HLA-ABC (Class I histocompatibility Antigen) were expressed to levels $>93.8 \%$ in the FS-hiPSC-MSCs. Another MSC surface marker, CD105, was expressed to greater than $65 \%$. On the other hand, the expressions of hematopoietic marker, CD31 and CD34, were less than $0.6 \%$ in the FS-hiPSC-MSCs. hESC pluripotency markers, HLA-DR (Class II histocompatibility Antigen) and TRA-1-81, were less than $0.2 \%$. These results showed that typical MSC markers were highly expressed in the FS-iPSC-MSCs. Fig. S1 shows representative phase contrast microscope figures of the hDPSCs, BM-hiPSC-MSCs, FS-hiPSC-MSCs and hBMSCs. The morphology of the cells appeared similar. They all exhibited a fibroblast-like morphology, and a small cell body with several cell processes that were long and thin.

Fig. 2 shows the physical properties of CPC-CAF constructs (mean $\pm \mathrm{SD} ; n=6$ ). In Fig. 2A, CN imparted fast-setting to CPC paste $(p<0.05)$. Setting time of CPC-CN-CAF-SU was about $7 \mathrm{~min}$, which was much faster than 19 min of CPC control $(p<0.05)$. Fig. 2B indicated CAF incorporation significantly increased the porosity of CPC $(p<0.05)$, which would be beneficial to tissue regeneration. The porosity of CPC-CN-CAF-SU was approximate $62 \%$, compared to $38 \%$ of CPC control $(p<0.05)$. Fig. $2 \mathrm{C}$ showed the external surface of the CPC scaffold, exhibiting uniformly mixed CPC particles. Selected specimens were cross-sectioned to reveal the interior of the scaffold. Typical pores and hydrogel fibers in the cross-section are shown in Fig. 2D. The yellow arrows indicate the micropores with diameter of a few tens of microns. The red arrows indicate the hydrogel fiber pullout from the CPC matrix. This 
suggests that hydrogel fibers were well mingled with the CPC paste during mixing, and macropores in the form of long channels would be created upon hydrogel fiber degradation.

Fig. 3 shows hDPSCs, BM-hiPSC-MSCs, FS-hiPSC-MSCs and hBMSCs encapsulated in hydrogel fibers without $\mathrm{CPC}$ and without injection, and those mixed in CPC paste and injected: (A-H) cell live/dead staining images, (I) percentage of live cells, and (J) live cell density (mean $\pm \mathrm{SD} ; n=6$ ). Live/dead staining images showed little difference between CAF without injection and after injection, indicating that the CPC paste mixing and injection did not harm the cells. In addition, there was no noticeable difference between the four types of cells. This was verified by the quantitative measurements in (I) and (J), where each horizontal line indicated values that were not significantly different from each other $(p>0.1)$.

The release of encapsulated cells is shown in Fig. 4. Fig. 4 (A-D) shows representative images of fluorescent live/dead staining for BM-hiPSC-MSCs; images for the other three types of cells were similar (Fig. S2). At 1 d, cells were located inside the hydrogel fibers and there was no cell release from the fibers. At $4 \mathrm{~d}$, CAF degradation was noticeable and there were some released cells around the fibers. At $7 \mathrm{~d}$, the hydrogel fibers appeared to be disintegrated and more cells were released and proliferated rapidly. At $14 \mathrm{~d}$, cell proliferation formed a monolayer covering CPC. At all the time points tested, there were numerous live cells (green staining) and only a few dead ones (red staining). The quantification of viability of cells released from the degrading hydrogel fibers in CPC is plotted in Fig. 4: (E) Percentage of live cells, (F) live cell density, and (G) cell proliferation measured via CCK-8 (mean $\pm \mathrm{sd} ; n=6$ ). In $(\mathrm{E})$, the percentages of live cells in the four groups were all above $90 \%$ and increased from $1 \mathrm{~d}$ to $14 \mathrm{~d}(p<0.05)$. In $(\mathrm{F})$, live cell density on CPC increased by 
10-fold from 1 to $14 \mathrm{~d}$. For hDPSCs, the live cell density was significantly higher than BM-hiPSC-MSCs, FS-hiPSC-MSCs, hBMSCs at $14 \mathrm{~d}(p<0.05)$. In $(\mathrm{G})$, hDPSCs showed greater proliferation on CPC than the other cells, with a 15-fold increase from 1 to $14 \mathrm{~d}$. BM-hiPSC-MSCs, FS-hiPSC-MSCs and hBMSCs exhibited a 10-fold increase from 1 to 14 d.

Osteogenic gene expressions of the encapsulated cells in CPC-CAF are plotted in Fig. 5: (A) ALP, (B) RUNX2, (C) COL1, and (D) OC (mean \pm sd; $n=6$ ). Up-regulations of ALP, RUNX2, COL1 and OCN were observed in all groups as early as $4 \mathrm{~d}$. ALP and OC in hDPSCs continuously and dramatically increased from 1 to $14 \mathrm{~d}$, which were over 32-fold and 12-fold that of $1 \mathrm{~d}$, respectively. BM-hiPSC-MSCs, FS-hiPSC-MSCs and hBMSCs had lower peaks of ALP and OC at $14 \mathrm{~d}$, compared with hDPSCs $(p<0.05)$. RUNX2 and COL1 peaked at 7 or $14 \mathrm{~d}$, with no significant differences between hDPSCs and hBMSCs $(p>0.1)$. FS-hiPSC-MSCs showed the lowest peaks in all gene expressions among the four cell types.

Mineral synthesis by cells in CPC-CAF is shown in Fig. 6: (A-L) xylenol orange staining, and (M) ARS staining. (A-L) showed that red staining increased with time. In $(\mathrm{N})$, mineralization increased by about 6-fold from $1 \mathrm{~d}$ to $14 \mathrm{~d}$ for hDPSCs, BM-hiPSC-MSCs and hBMSCs $(p<0.05)$. FS-hiPSC-MSCs showed only 3-fold increase in mineralization from $1 \mathrm{~d}$ to $14 \mathrm{~d}(p<0.05)$, significantly less than the other groups $(p<0.05)$. ARS staining of the synthesized bone mineral became denser and darker over time (Fig. 6M). The mineral concentration increased by 14 folds for hDPSCs, BM-hiPSC-MSCs and hBMSCs, versus 8 folds for FS-hiPSC-MSCs from 1 to $14 \mathrm{~d}$ (Fig. 6O). FS-hiPSC-MSCs had the lowest mineral concentration $(p<0.05)$ (Fig. 6O). For both $(\mathrm{N})$ and $(\mathrm{O})$, at each time period, there was no 
significant difference among hDPSCs, BM-hiPSC-MSCs and hBMSCs $(p>0.1)$. In order to investigate whether such differences were caused by the various osteogenic potentials of the four types of cells, we detected mineral synthesis of the cells before encapsulation (Fig. S3). After $7 \mathrm{~d}$ osteogenic induction, there were no significant differences in mineralization for the four cell types (Fig. S3). However, after 14 d osteogenic induction, FS-hiPSC-MSC group exhibited less mineralized nodules than the other three types of cells, indicating that FS-hiPSC-MSC had relatively inferior osteogenic capability than the other cells tested. hDPSCs showed the highest level of mineralized area percentage and mineral concentration among the four groups at $14 \mathrm{~d}$ (Fig. S3). These results further confirmed that the four MSCs from different tissues indeed exhibited different osteogenic potential.

\section{Discussion}

The present study derived MSCs from hiPSCs from two different origins (bone marrow and foreskin), and compared hDPSCs, BM-hiPSC-MSCs, FS-hiPSC-MSCs and hBMSCs encapsulated in hydrogel fibers in an injectable CPC construct for the first time. The alginate-fibrin fibers were shown to be suitable for cell encapsulation, and protecting cells during CPC paste mixing and injection process. The injectable CPC, reinforced by chitosan and degradable suture, had good affinity for cell attachment and showed no negative effect on cell viability after injection. All four types of stem cells underwent osteogenic differentiation in CPC. Nevertheless, FS-iPSC-MSCs were inferior in osteogenic differentiation than the other three cells. Therefore, this study showed that hDPSCs and BM-hiPSC-MSCs were viable alternative cells to the gold-standard hBMSCs, while FS-iPSC-MSCs appeared to be 
inferior as seeding cells for bone repair.

$\mathrm{CPC}$ is an injectable bone cement and can set in situ to form a bioactive and osteoconductive scaffold that bonds to bone [42]. Previous studies indicated that chitosan rendered CPC fast-setting and mechanically strong [38]. In the present study, CAF-CN-SU shortened the setting time from $19 \mathrm{~min}$ to $7 \mathrm{~min}$. Such fast setting should enable the graft to attain significant strength and geometrical integrity within a short time during surgery. The rapid setting could improve the initial mechanical strength, thereby avoiding implant disintegration and accompanied inflammation [43]. To boost its bone formation efficiency, the present study developed an injectable, bioactive and macroporous CPC scaffold for stem cell encapsulation and bone tissue engineering. A fast-degradable alginate-fibrin hydrogel was used to encapsulate cells in CPC. The purpose of the hydrogel fibers was to protect cell viability during CPC mixing, injection and setting reaction, as shown by the live/dead staining results. SEM examination showed that the hydrogel fibers were relatively evenly distributed in CPC. After CPC setting, the fast-degradable hydrogel fibers would start to degrade and release the cells throughout the entire CPC construct. Upon degradation, the hydrogel fibers would create long macropore channels to increase the porosity and pore interconnectivity of CPC. The interconnected macropores would be beneficial to allow oxygen and nutrients to reach the cells in the scaffold, and provide more space for cells to communicate with each other in CPC. Furthermore, the methodology of combining the cell carrier hydrogel fibers with macropores is expected to benefit vascular ingrowth in the new bone. During CPC setting, CPC undergoes a phase conversion from TTCP and DCPA to apatite, as previously verified by XRD [44]. In addition, previous studies showed that the 
encapsulated hBMSCs and human umbilical cord mesenchymal stem cells (hUCMSCs) in hydrogel microbeads successfully synthesized bone minerals inside the CPC scaffold [45]. SEM and XRD examinations confirmed that the cell-synthesized minerals were a low-crystalline apatite similar to bone minerals [45]. These initial in vitro results are promising and warrant further animal studies to evaluate the in vivo osteogenesis and angiogenesis via stem cell-CPC constructs.

In the present study, hDPSCs exhibited high proliferation, high gene expression of osteogenic markers and cell mineralization. Previous studies reported that hDPSCs exhibited a similar gene expression profile to hBMSCs [46], and higher proliferation rate than hBMSCs [20]. It was also reported that DPSCs produced three-dimensional woven bone-like structure in vitro, which could be remodeled to lamellar bone containing entrapped osteocytes in vivo $[22,47]$. These findings, along with the present results, indicated that hDPSCs appeared to be a promising alternative to hBMSCs in bone tissue engineering.

The recent discovery of iPSCs marked a major breakthrough for stem cells and regenerative medicine. With the potential to develop into many cell types, iPSCs are regarded as the new frontier for cell therapy and cell-based tissue engineering. Both the BM-hiPSCs and FS-hiPSCs used in the present study had been extensively tested in previous studies which demonstrated that these iPS cell lines had normal karyotypes, expressed cell surface markers that characterized human ES cells, and maintained the developmental potential to differentiate into advanced derivatives of all three primary germ layers $[48,49]$. These previous studies indicated that both these iPS cell lines were generated properly $[48,49]$.

However, it is reported that iPSCs retain, at least transiently, epigenetic memory related 
to the donor cell of origin, which may affect their subsequent differentiation potential [16-19]. In other words, the tendency of early-passage iPSC lines is to differentiate preferentially into the cell lineage of origin [19]. In the present study, BM-hiPSC-MSCs showed higher osteogenic gene expression and mineral deposition than FS-hiPSC-MSCs, which supported the epigenetic memory theory. To date, a few studies have investigated iPSC cell lines arising from different somatic cell sources and compared the in vitro and in vivo osteogenic potentials. In one study, three hiPSC cell lines (11c, 1013A both derived from dermal fibroblasts, and $\mathrm{BC} 1$ derived from bone marrow cells) were induced into mesenchymal lineage [50]. When seeded on an osteo-conductive scaffold, the BC1-iPSC-MSC-engineered bone constructs displayed regions with the highest density of cells and new bone-like matrix in vitro perfusion culture among the three iPSC-MSC cell seeding constructs [50]. Adversely, the 11c-derived progenitors exhibited lower antigenic expression and poorer osteogenic differentiation as revealed by ALP activity and calcium deposition than the other two cell lines [50]. Upon in vivo subcutaneous implantation into immunodeficient mice for 12 weeks, bone constructs engineered using $\mathrm{BC} 1$-iPSC-MSCs displayed higher mineral content compared with iPSC-MSCs derived from fibroblasts [50]. Another study reported that iPSCs from human deciduous teeth stem cell (SHED) exhibited significantly higher osteogenic potential than iPSCs from dermal fibroblasts via an in vitro osteogenesis comparison [51]. Hynes et al. generated MSC-like cells from iPSC lines arising from three different somatic tissues: gingiva, periodontal ligament (PDL) and lung, respectively [52]. Although they all displayed multi-lineage differentiation capacity in the in vitro assays, only PDL-derived iPSC-MSC-like cells formed mature mineralized structures in vivo when mixed with HA/TCP 
particles [52].

However, not all studies reflected the above-mentioned epigenetic memory theory. One study compared the bone forming ability of iPSC-MSCs derived from two distinct tissue sources: human skin fibroblasts (SF) and BMSCs. The two iPSC-MSC cell lines were seeded on the HA/TCP scaffolds and subcutaneously implanted into immunocompromised mice. Bone of equal quality and frequency was made from both SF- or BMSC-derived iPSC-MSCs, suggesting that iPSCs did not retain any 'memory' of their origin [53]. Similarly, another research generated rhesus iPSCs from rhesus BMSCs (rhBMSCs), skin fibroblasts, and CD34+ cells and then differentiated the iPSCs into MSC-like cells before seeding on HA/TCP particles [54]. When the constructs were implanted subcutaneously in the autologous macaques, robust bone formation was observed in all grafts regardless of the iPSC origins. Further studies are needed to investigate and clarify this issue.

\section{Conclusions}

This study developed a novel injectable CPC-hydrogel fiber-stem cell construct and compared hDPSCs, BM-hiPSC-MSCs, FS-hiPSC-MSCs and hBMSCs inside CPC for bone engineering for the first time. The injectable scaffold was fast-setting and highly porous. The injection did not harm the encapsulated cells. hDPSCs, BM-hiPSC-MSCs, FS-hiPSC-MSCs and hBMSCs in CPC-CAF construct exhibited excellent proliferation. hDPSCs had the highest proliferative rate. hDPSCs, BM-hiPSC-MSCs and hBMSCs in CPC-CAF differentiated into the osteogenic lineage, with highly elevated ALP, RUNX2, COL1A1 and OC. Mineralization of hDPSCs, BM-hiPSC-MSCs and hBMSCs in scaffolds substantially 
increased over time. The results showed that hDPSCs, BM-hiPSC-MSCs and hBMSCs were all promising cell sources for bone engineering; however, FS-hiPSC-MSCs were relatively inferior for osteogenic differentiation. The novel injectable CPC with cell-encapsulating hydrogel fibers is promising to enhance bone regeneration in dental, craniofacial and orthopedic applications.

\section{Acknowledgements}

We thank Prof. Linzhao Cheng of Johns Hopkins University for providing the hiPSC BC1 cells, and Prof. Ashraf F. Fouad of University of Maryland, School of Dentistry for providing the hDPSCs. This study was supported by NIH R01 DE14190 and R21 DE22625 (HX), National Science Foundation of China 81400487 (LW) and 81401794 (PW), Youth Fund of Science and Technology of Jilin Province 20150520043JH (LW), China Postdoctoral Science Foundation 2015M581405 (LW), China Scholarship Council (LW), and University of Maryland School of Dentistry bridge fund (HX). There is no conflict of interest.

\section{Reference}

[1] J.D. Kretlow, A.G. Mikos, Review: mineralization of synthetic polymer scaffolds for bone tissue engineering, Tissue Eng. 13 (2007) 927-938.

[2] A.J. Salgado, O.P. Coutinho, R.L. Reis, Bone tissue engineering: state of the art and future trends, Macromol. Biosci. 4 (2004) 743-765.

[3] A.J. Salgado, J.T. Oliveira, A.J. Pedro, R.L. Reis, Adult stem cells in bone and cartilage tissue engineering, Curr. Stem Cell Res. T. 1 (2006) 345-364. 
[4] X. Xin, M. Hussain, J.J. Mao, Continuing differentiation of human mesenchymal stem cells and induced chondrogenic and osteogenic lineages in electrospun PLGA nanofiber scaffold, Biomaterials 28 (2007) 316-325.

[5] H. Park, J.S. Temenoff, Y. Tabata, A.I. Caplan, A.G. Mikos, Injectable biodegradable hydrogel composites for rabbit marrow mesenchymal stem cell and growth factor delivery for cartilage tissue engineering, Biomaterials 28 (2007) 3217-3227.

[6] G. Kumar, C.K. Tison, K. Chatterjee, P.S. Pine, J.H. McDaniel, M.L. Salit, et al., The determination of stem cell fate by 3D scaffold structures through the control of cell shape, Biomaterials 32 (2011) 9188-9196.

[7] G.C. Reilly, S. Radin, A.T. Chen, P. Ducheyne, Differential alkaline phosphatase responses of rat and human bone marrow derived mesenchymal stem cells to 45S5 bioactive glass, Biomaterials 28 (2007) 4091-4097.

[8] J. Mao, G. Vunjak-Novakovic, A. Mikos, A. Atala, Regenerative medicine: Translational approaches and tissue engineering, Boston, MA: Artech House (2007) 1-3.

[9] D. Baksh, R. Yao, R.S. Tuan, Comparison of proliferative and multilineage differentiation potential of human mesenchymal stem cells derived from umbilical cord and bone marrow, Stem Cells 25 (2007) 1384-1392.

[10] A.F. Steinert, L. Rackwitz, F. Gilbert, U. Nöth, R.S. Tuan, Concise review: the clinical application of mesenchymal stem cells for musculoskeletal regeneration: current status and perspectives, St Stem Cells Transl. Med. 1 (2012) 237-247.

[11] B.S. Shah, P.A. Clark, E.K. Moioli, M.A. Stroscio, J.J. Mao, Labeling of mesenchymal stem cells by bioconjugated quantum dots, Nano letters 7 (2007) 3071-3079. 
[12] K. Takahashi, K. Tanabe, M. Ohnuki, M. Narita, T. Ichisaka, K. Tomoda, et al., Induction of pluripotent stem cells from adult human fibroblasts by defined factors, Cell 131 (2007) 861-872.

[13] P.S. Knoepfler, Deconstructing stem cell tumorigenicity: a roadmap to safe regenerative medicine, Stem Cells 27 (2009) 1050-1056.

[14] P. Wang, X. Liu, L. Zhao, M.D. Weir, J. Sun, W. Chen, et al., Bone tissue engineering via human induced pluripotent, umbilical cord and bone marrow mesenchymal stem cells in rat cranium, Acta Biomater. 18 (2015) 236-248.

[15] H. Wei, G. Tan, S. Qiu, G. Kong, P. Yong, C. Koh, et al., One-step derivation of cardiomyocytes and mesenchymal stem cells from human pluripotent stem cells, Stem Cell Res 9 (2012) 87-100.

[16] K. Kim, A. Doi, B. Wen, K. Ng, R. Zhao, P. Cahan, et al., Epigenetic memory in induced pluripotent stem cells, Nature 467 (2010) 285-290.

[17] K. Kim, R. Zhao, A. Doi, K. Ng, J. Unternaehrer, P. Cahan, et al., Donor cell type can influence the epigenome and differentiation potential of human induced pluripotent stem cells, Nat. Biotechol. 29 (2011) 1117-1119.

[18] O. Bar-Nur, H.A. Russ, S. Efrat, N. Benvenisty, Epigenetic memory and preferential lineage-specific differentiation in induced pluripotent stem cells derived from human pancreatic islet beta cells, Cell Stem Cell 9 (2011) 17-23.

[19] J.M. Polo, S. Liu, M.E. Figueroa, W. Kulalert, S. Eminli, K.Y. Tan, et al., Cell type of origin influences the molecular and functional properties of mouse induced pluripotent stem cells, Nat. Biotechnol. 28 (2010) 848-855. 
[20] S. Gronthos, M. Mankani, J. Brahim, P.G. Robey, S. Shi, Postnatal human dental pulp stem cells (DPSCs) in vitro and in vivo, P. Natl. Acad. Sci. USA 97 (2000) 13625-13630.

[21] S. Gronthos, J. Brahim, W. Li, L. Fisher, N. Cherman, A. Boyde, et al., Stem cell properties of human dental pulp stem cells, J. Dent Res. 81 (2002) 531-535.

[22] G. Laino, R. d'Aquino, A. Graziano, V. Lanza, F. Carinci, F. Naro, et al., A new population of human adult dental pulp stem cells: a useful source of living autologous fibrous bone tissue (LAB), J. Bone Miner. Res. 20 (2005) 1394-1402.

[23] P. Ducheyne, Q. Qiu, Bioactive ceramics: the effect of surface reactivity on bone formation and bone cell function, Biomaterials 20 (1999) 2287-2303.

[24] L. Zhao, M.D. Weir, H.H. Xu, An injectable calcium phosphate-alginate hydrogel-umbilical cord mesenchymal stem cell paste for bone tissue engineering, Biomaterials 31 (2010) 6502-6510.

[25] J.D. Kretlow, S. Young, L. Klouda, M. Wong, A.G. Mikos, Injectable biomaterials for regenerating complex craniofacial tissues, Adv. Mater. 21 (2009) 3368-3393.

[26] N.A. Raof, M.R. Padgen, A.R. Gracias, M. Bergkvist, Y. Xie, One-dimensional self-assembly of mouse embryonic stem cells using an array of hydrogel microstrands, Biomaterials 32 (2011) 4498-4505.

[27] L. Cheng, N.F. Hansen, L. Zhao, Y. Du, C. Zou, F.X. Donovan, et al., Low incidence of DNA sequence variation in human induced pluripotent stem cells generated by nonintegrating plasmid expression, Cell Stem Cell 10 (2012) 337-344.

[28] J. Liu, W. Chen, Z. Zhao, H.H. Xu, Reprogramming of mesenchymal stem cells derived from iPSCs seeded on biofunctionalized calcium phosphate scaffold for bone engineering, 
Biomaterials 34 (2013) 7862-7872.

[29] M. Tang, W. Chen, M.D. Weir, W. Thein-Han, H.H. Xu, Human embryonic stem cell encapsulation in alginate microbeads in macroporous calcium phosphate cement for bone tissue engineering, Acta Biomater. 8 (2012) 3436-3445.

[30] D.J. Alongi, T. Yamaza, Y. Song, A.F. Fouad, E.E. Romberg, S. Shi, et al., Stem/progenitor cells from inflamed human dental pulp retain tissue regeneration potential, Regen. Med. 5 (2010) 617-631.

[31] K.S. Anseth, C.N. Bowman, L. Brannon-Peppas, Mechanical properties of hydrogels and their experimental determination, Biomaterials 17 (1996) 1647-1657.

[32] K.H. Bouhadir, K.Y. Lee, E. Alsberg, K.L. Damm, K.W. Anderson, D.J. Mooney, Degradation of partially oxidized alginate and its potential application for tissue engineering, Biotechnol. Progr. 17 (2001) 945-950.

[33] H. Zhou, H.H. Xu, The fast release of stem cells from alginate-fibrin microbeads in injectable scaffolds for bone tissue engineering, Biomaterials 32 (2011) 7503-7513.

[34] S.M. Mihaila, E.G. Popa, R.L. Reis, A.P. Marques, M.E. Gomes, Fabrication of endothelial cell-laden carrageenan microfibers for microvascularized bone tissue engineering applications, Biomacromolecules 15 (2014) 2849-2860.

[35] K. HoáLee, Novel PDMS cylindrical channels that generate coaxial flow, and application to fabrication of microfibers and particles, Lab Chip 10 (2010) 1856-1861.

[36] A.R. Costa-Pinto, R.L. Reis, N.M. Neves, Scaffolds based bone tissue engineering: the role of chitosan, Tissue Eng. Part B 17 (2011) 331-347.

[37] P. Wang, Y. Song, M.D. Weir, J. Sun, L. Zhao, C.G. Simon, et al., A self-setting 
iPSMSC-alginate-calcium phosphate paste for bone tissue engineering, Dent. Mater. 32 (2016) 252-263.

[38] H. Xu, S. Takagi, J. Quinn, L. Chow, Fast-setting and anti-washout calcium phosphate scaffolds with high strength and controlled macropore formation rates, J. Biomed. Mater. Res. A 68 (2004) 725-734.

[39] W. Chen, H. Zhou, M.D. Weir, C. Bao, H.H. Xu, Umbilical cord stem cells released from alginate-fibrin microbeads inside macroporous and biofunctionalized calcium phosphate cement for bone regeneration, Acta Biomater. 8 (2012) 2297-2306.

[40] Y. Shi, J.R. Niedzinski, A. Samaniego, S. Bogdansky, B.L. Atkinson, Adipose-derived stem cells combined with a demineralized cancellous bone substrate for bone regeneration, Tissue Eng. Part A 18 (2012) 1313-1321.

[41] Y.H. Wang, Y. Liu, P. Maye, D.W. Rowe, Examination of mineralized nodule formation in living osteoblastic cultures using fluorescent dyes, Biotechnol. Progr. 22 (2006) $1697-1701$.

[42] M. Bohner, G. Baroud, Injectability of calcium phosphate pastes, Biomaterials 26 (2005) $1553-1563$.

[43] L.E. Carey, H.H. Xu, C.G. Simon, S. Takagi, L.C. Chow, Premixed rapid-setting calcium phosphate composites for bone repair, Biomaterials 26 (2005) 5002-5014.

[43] M.N. Rahaman, D.E. Day, B.S. Bal, Q. Fu, S.B. Jung, L.F. Bonewald, et al., Bioactive glass in tissue engineering, Acta Biomater. 7 (2011) 2355-2373.

[44] M.D. Weir, H.H. Xu, Human bone marrow stem cell-encapsulating calcium phosphate scaffolds for bone repair, Acta Biomater. 6 (2010) 4118-4126. 
[45] L. Zhao, M.D. Weir, H.H. Xu, Human umbilical cord stem cell encapsulation in calcium phosphate scaffolds for bone engineering, Biomaterials 31 (2010) 3848-3857.

[46] S. Shi, P. Robey, S. Gronthos, Comparison of human dental pulp and bone marrow stromal stem cells by cDNA microarray analysis, Bone 29 (2001) 532-539.

[47] G. Laino, A. Graziano, R. d'Aquino, G. Pirozzi, V. Lanza, S. Valiante, et al., An approachable human adult stem cell source for hard-tissue engineering, J. Cell. Physiol. 206 (2006) 693-701.

[48] J. Yu, M.A. Vodyanik, K. Smuga-Otto, J. Antosiewicz-Bourget, J.L. Frane, S. Tian, et al., Induced pluripotent stem cell lines derived from human somatic cells, Science 318 (2007) 1917-1920.

[49] B.K. Chou, P. Mali, X. Huang, Z. Ye, S.N. Dowey, L.M. Resar, et al., Efficient human iPS cell derivation by a non-integrating plasmid from blood cells with unique epigenetic and gene expression signatures, Cell Res. 21 (2011) 518-529.

[50] G.M. de Peppo, I. Marcos-Campos, D.J. Kahler, D. Alsalman, L. Shang, G. Vunjak-Novakovic, et al., Engineering bone tissue substitutes from human induced pluripotent stem cells, P. Natl. Acad. Sci. USA 110 (2013) 8680-8685.

[51] F.A.A. Ishiy, R.D. Fanganiello, K. Griesi-Oliveira, A.M. Suzuki, G.S. Kobayashi, A.G. Morales, et al., Improvement of In Vitro Osteogenic Potential through Differentiation of Induced Pluripotent Stem Cells from Human Exfoliated Dental Tissue towards Mesenchymal-Like Stem Cells, Stem Cells Int. 2015 (2015) 249098.

[52] K. Hynes, D. Menicanin, K. Mrozik, S. Gronthos, P.M. Bartold, Generation of functional mesenchymal stem cells from different induced pluripotent stem cell lines, Stem Cells Dev. 
23 (2013) 1084-1096.

[53] M.D. Phillips, S.A. Kuznetsov, N. Cherman, K. Park, K.G. Chen, B.N. McClendon, et al., Directed differentiation of human induced pluripotent stem cells toward bone and cartilage: in vitro versus in vivo assays, Stem Cells Transl. Med. 3 (2014) 867-878.

[54] S.G. Hong, T. Winkler, C. Wu, V. Guo, S. Pittaluga, A. Nicolae, et al., Path to the clinic: assessment of iPSC-based cell therapies in vivo in a nonhuman primate model, Cell Rep. 7 (2014) 1298-1309.

\section{Figure captions:}

[1] Immunophenotyping of human iPSC (Foreskin)-MSCs. Flow cytometry showed that FS-iPSC-MSCs expressed a number of cell surface markers characteristic of MSCs, and were negative for pluripotent, typical hematopoietic and endothelial cell markers. The names of the antigens are listed inside each plot. Each plot represents the percentage of positive cells. For example, MSC surface markers CD29, CD44, CD166 and CD73 were expressed to levels greater than 90\%, while expressions of hematopoietic markers (CD31 and CD34) were less than $0.6 \%$.

[2] Physical properties of CPC-CAF construct: (A) paste setting time, and (B) porosity (mean $\pm \mathrm{sd} ; \mathrm{n}=6$ ). $\mathrm{CAF}$ : cell-encapsulating alginate-fibrin fibers. $\mathrm{CN}$ : chitosan. $\mathrm{SU}$ : suture fibers. In each plot, bars indicated by different letters are significantly different $(\mathrm{p}<0.05)$. (C, D) Representative SEM images of the CPC scaffold containing alginate-fibrin microfibers. (C) The external surface of the CPC specimen showed uniform structure with well mixed CPC particles. (D) The crossing section of CPC specimens showed pores 
(yellow arrows) and hydrogel fibers (red arrows). Dissimilar letters indicate significantly different values $(\mathrm{p}<0.05)$.

[3] Cells viability without injection and after injection: (A-D) Cells in CAF (without CPC, without injection), and (E-H) cells in $\mathrm{CAF}$ after mixing with $\mathrm{CPC}$ paste and after injection. Live cells (green) were numerous. Dead cells (red) were few. (I): Percentage of live cells, and (J) live cell density (mean $\pm \mathrm{sd} ; n=6$ ). The CAF protected the cells, yielding viability after CPC mixing/injection to be similar to that without injection $(p>0.1)$. Horizontal line indicates values that are statistically similar $(p>0.1)$.

[4] Cell release and proliferation from CAF for hDPSCs, BM-hiPSC-MSCs, FS-hiPSC-MSCs and hBMSCs: (A-D) live/dead staining and fluorescence microscopy of BM-hiPSC-MSCs released to CPC surface. At $4 \mathrm{~d}$, cells started to be released from fibers. From 7 to $14 \mathrm{~d}$, more cells were released and the contours of fibers became obscure as they degraded. Live/dead images in (A-D) showed numerous live cells with few dead cells, and the live cell numbers greatly increased from 1 to $14 \mathrm{~d}$ for all cell types. (E) Percentage of live cells, (F) live cell density, and (G) CCK-8 cell proliferation (mean $\pm \mathrm{sd}$; $n=6$ ).

[5] Osteogenic differentiation of hDPSCs, BM-hiPSC-MSCs, FS-hiPSC-MSCs and hBMSCs in CPC-CAF constructs: (A) Alkaline phosphatase activity (ALP), (B) Runt-related transcription factor 2 (RUNX2), (C) collagen type I (COL1A1), (D) osteocalcin (OC) gene expressions (mean $\pm \mathrm{sd} ; n=6$ ). Cells cultured for $1 \mathrm{~d}$ served as the calibrator. In each plot, values with dissimilar letters are significantly different with each other $(p<0.05)$. Dissimilar letters indicate significantly different values $(\mathrm{p}<0.05)$.

[6] Synthesis of bone minerals by encapsulated stem cells. Xylenol orange mineral staining 
images of (A-C) hDPSCs, (D-F) BM-hiPSC-MSCs, (G-I) FS-hiPSC-MSCs, and (J-L) hBMSCs. (M) ARS staining images of hDPSCs, BM-hiPSC-MSCs, FS-hiPSC-MSCs and hBMSCs in CPC-CAF. (N) Xylenol orange mineral staining area fraction (mean $\pm \mathrm{sd} ; n=6$ ). (O) ARS mineral concentration synthesized by cells in CPC-CAF measured by an osteogenesis assay (mean $\pm \mathrm{sd} ; n=6$ ).

[S1] Representative images of the four stem cells under phase contrast microscope showing fibroblast like morphology.

[S2] Live/dead staining of cell release from CAF: (A-D) hBMSCs, (E-H) hDPSCs, and (I-L) FO-hiPSC-MSCs. There were numerous live cells with few dead cells, and the live cell numbers greatly increased from $1 \mathrm{~d}$ to $14 \mathrm{~d}$ for all cell types.

[S3] Comparisons of the osteogenic potentials of the four stem cells pre-encapsulation. (A) Xylenol orange mineral staining. (B) ARS staining. (C). Xylenol orange mineral staining area fraction (mean $\pm \mathrm{sd} ; \mathrm{n}=6$ ). (O) ARS mineral concentration synthesized by cells (mean $\pm s d ; n=6)$. Dissimilar letters indicate significantly different values $(p<0.05)$. 
Figure 2
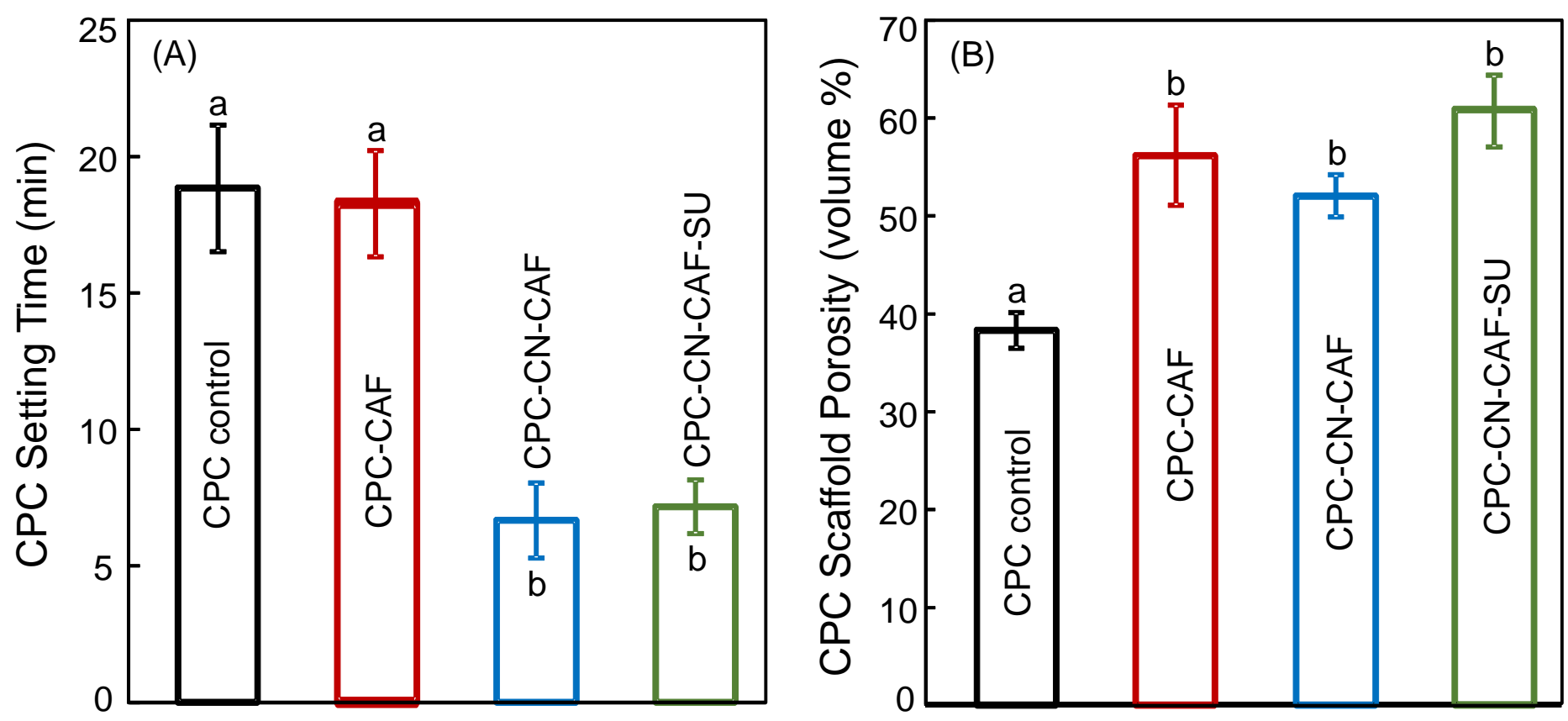

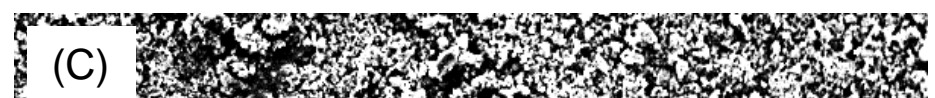

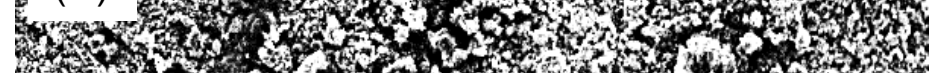
W.

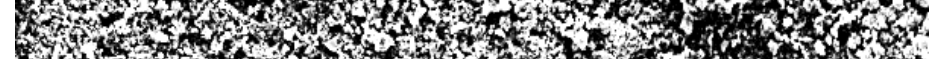

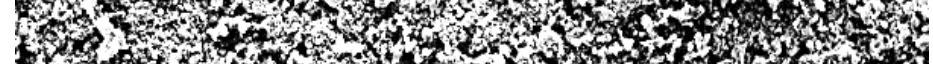
-7, W

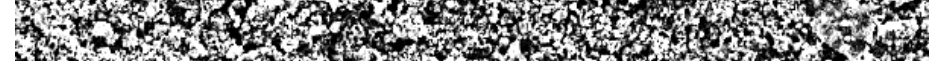

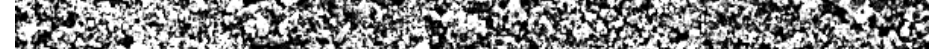

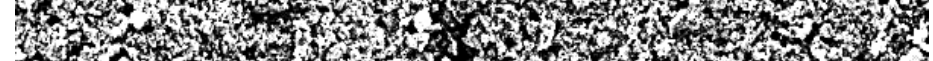

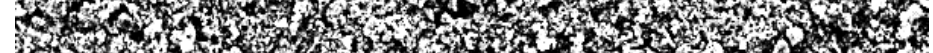

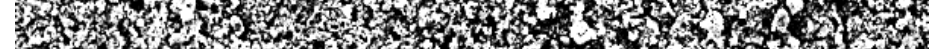

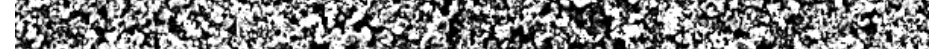

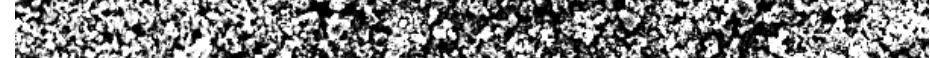

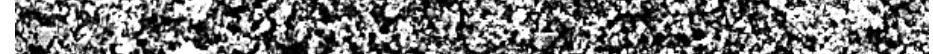

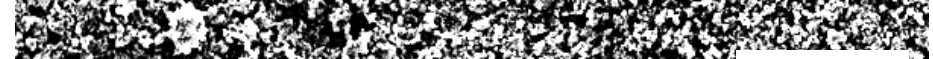

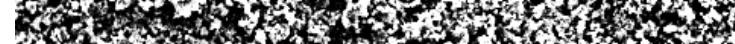

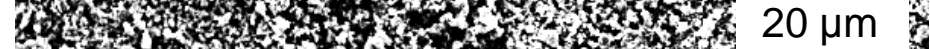
A

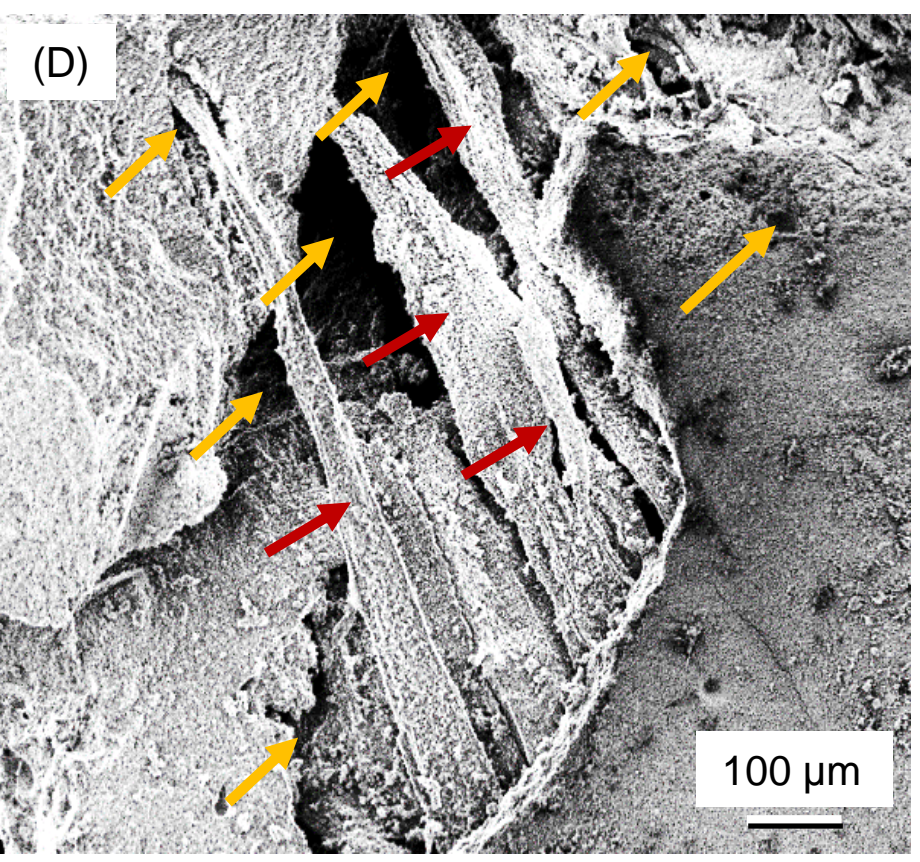

Fig. 2 

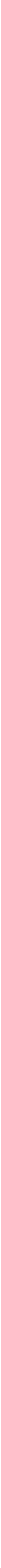

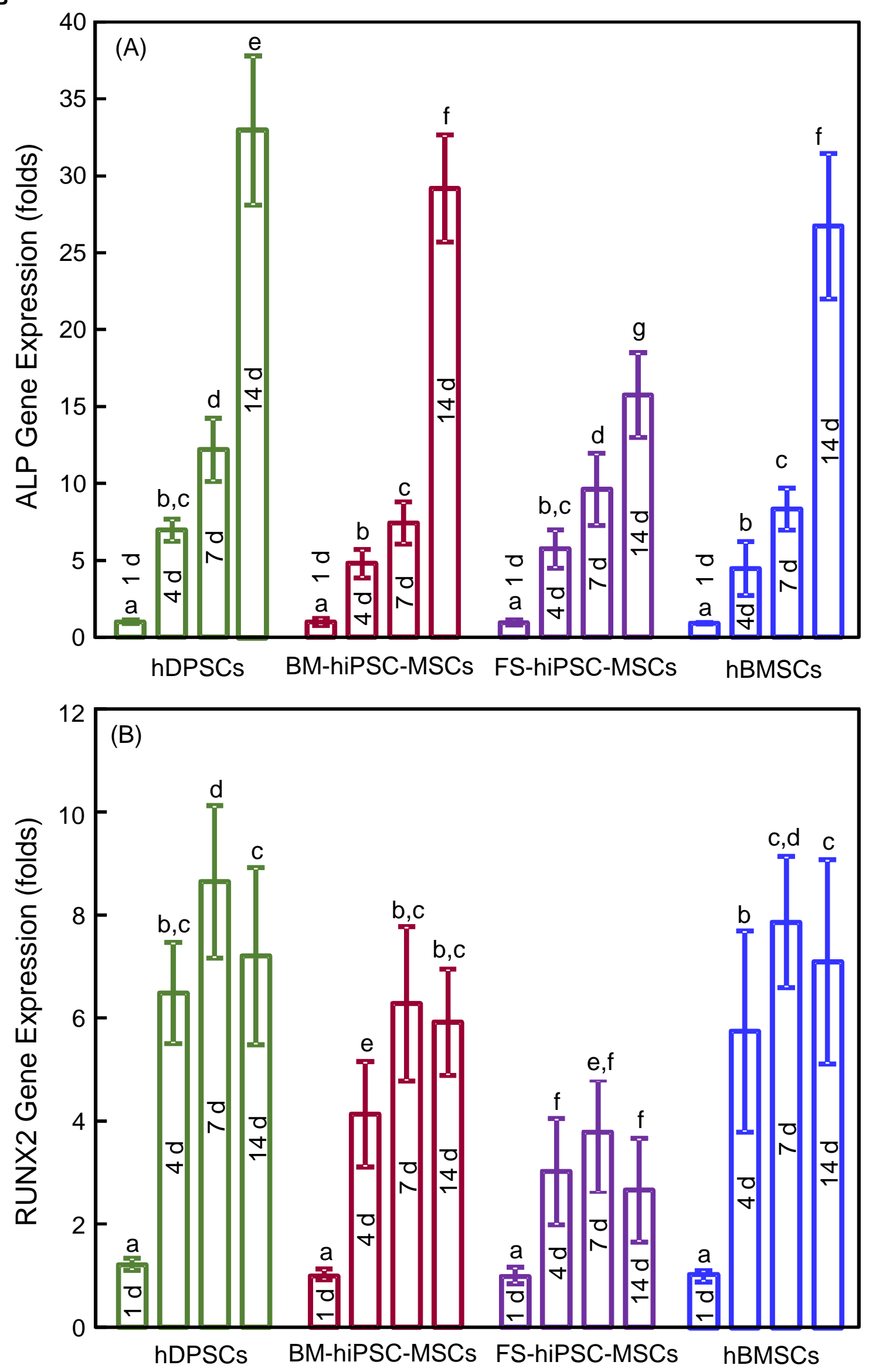

Fig. $5 \mathrm{~A}, 5 \mathrm{~B}$ 

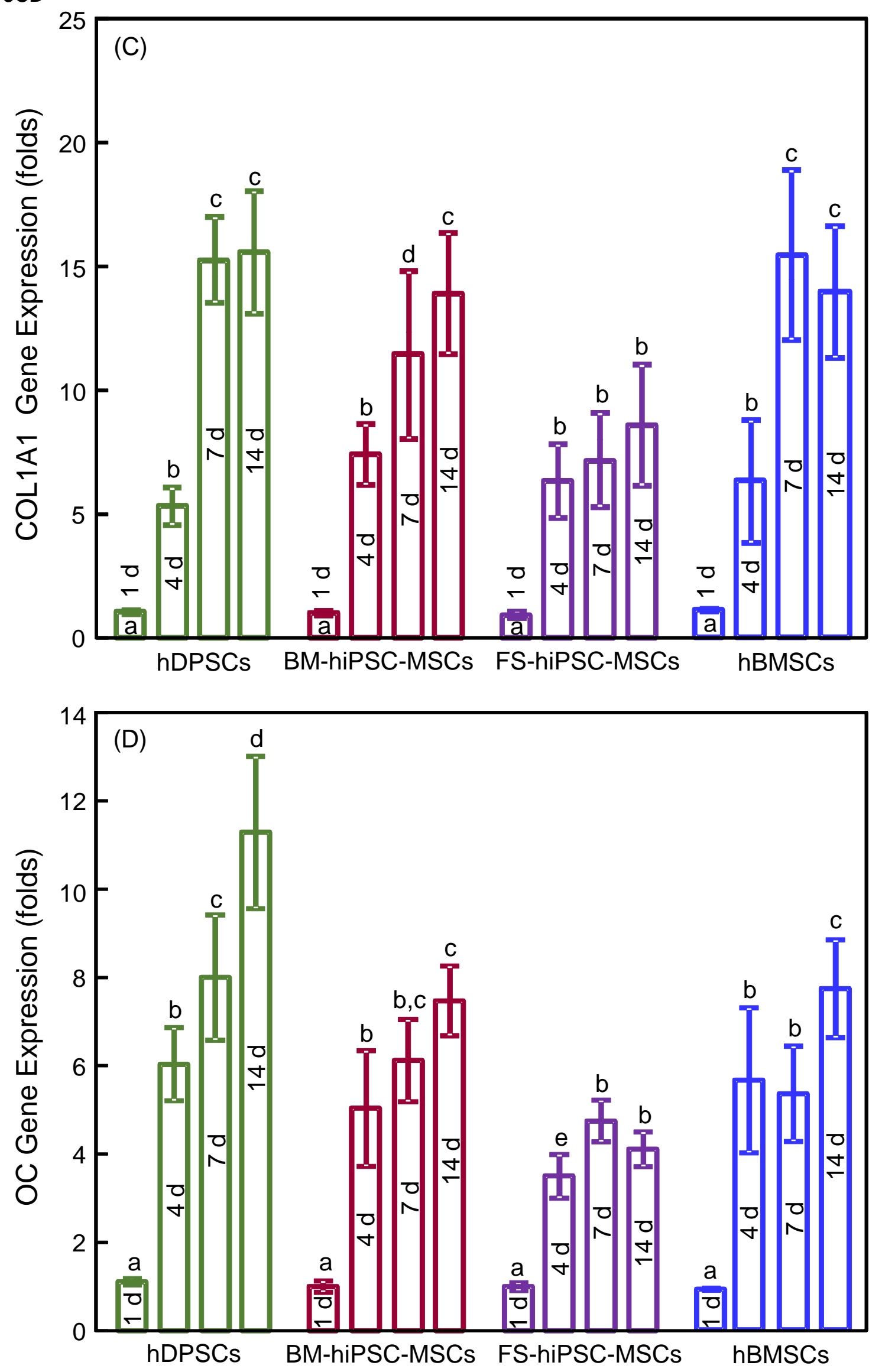

Fig. $5 \mathrm{C}, 5 \mathrm{D}$ 
